\title{
Exploring the problem of Internet Addiction: A Review and Analysis of Existing Literature
}

\author{
${ }^{1}$ Sumanjeet SINGH, ${ }^{2}$ Minakshi PALIWAL \\ 1. First Author \& Corresponding Author Dr. Ramjas College, University of Delhi, Delhi-7, INDIA. Email: \\ sumanjeetsingh@gmail.com \\ 2. Co-Author Dr. Daulat Ram College, University of Delhi, Delhi-7, INDIA
}

Received: March 07, 2020 Revised: March 17, 2020 Accepted: March 27, 2020

\begin{abstract}
Anecdotal reports indicate that maladaptive patterns of Internet use constitute behavioral addiction. Internet addiction is characterized by unrestrained and awfully controlled engrossment or behaviors regarding internet access that lead to impairment, stress, dimensionally measured depression, indicators of social separation and anguish. By reviewing and analyzing approximately 100 articles we present evidence that are able to provide an overview of the main themes and proclivity covered by existing and relevant studies. The vital detection of this research unveils that many factors related to social, demographic, lifestyle changes related constructs have a bearing on the phenomenon of internet addiction strongly. This study not only reached certain conclusions for both theory and practice, but also defined future lines of research according to the gaps detected by the study's results. The main findings from this literature, though not conclusive, but will help the researcher and policymakers to obtain a better understanding and description of the problem faced by the youth and necessary to develop some remedies to lessen the addiction phenomenon.
\end{abstract}

Keywords: Internet Addiction, Compulsive Behavioral, Disorder, Health

JEL Classification Code: E22, F24

\section{Introduction}

The upsurge and popularity of the internet as a communication and conveyance channel has become an ever-increasing part of many people's day-to-day lives. Incorporated with the rise in popularity and usage, there have been a increasing number of reports in the popular press about the excessive use of the internet under the guise of "Internet Addiction", "Internet Addiction Disorder" (IAD) and "Internet Addiction Syndrome"(IAS). Along with new technologies, the Internet has reshaped and upgraded many facets of our lives by being integrated in the daily experience, by becoming more available, offering many services and its growing usage in every age group. Internet Live Stats, data of internet users shows that over the last 15 years, the numbers of internet users have increased by $1000 \%$. That means approximately $46 \%$ of the world population is on the Internet today, an incredible growth compared to only less than $1 \%$ in 1995 (Internet Live Stats). At the beginning of the $21^{\text {st }}$ century, the transfiguration and development of the various life spheres occurred, due to the powerful influence of the internet. In December 2017, the number of internet users worldwide reached 4.15 billion (meaning an internet penetration of 54.4 per cent), up from 394 million in 2000 and 1 billion in 2005 (According to an estimate, more than 10 new people come onto the internet every second that 
translates to almost 1 million new users every day, according to Digital in 2017-Report (as cited in Kemp, 2017). There are 4.39 billion internet users in 2019, an increase of 366 million (9 percent) versus January 2018. There are 3.48 billion social media users in 2019, with the worldwide total growing by 288 million ( 9 percent) since this time last year (Internet Stats 2019), from 3.3 billion users, which is $46.4 \%$ of the world population (Internet World Stats, 2012).

The past decade has seen advances in mobile and other wearable technologies developing ubiquitous connection of individuals to the online world, being the most important factors driving internet addiction-IA (Turel \& Serenko 2010). Considering that the internet in as integral part of social and professional life, it is necessary to understand the fundamental reasons for its use and understanding the ambiguity of its impact and aspects of both positive and negative social consequences that provokes researchers, from different areas to the problem (Arshinova \& Bartsalkina, 2010; Gubanov \& Chkhartishvili, 2009; Khil'ko, 2015; Khutornoi, 2013). Methodical and comprehensive research on internet addiction indicated that it is associated with various risk factors namely sociodemographic factors like specific gender, younger age,people who spend more time online and on gaming along with many psychological factors including loneliness and impulsivity. This all suggest that these factors contribute to an increased vulnerability for developing internet-use related problems.

Studies on internet highlight that internet use can help improve results on tests and expand and increase motivation for learning among various groups of people, especially young people (Guan \& Subrahmanyam 2009). Also, perceived quality of life can be affected in the absence of Internet access (Pontes et al., 2015; Cao, Sun, \& Wan, 2011). While the use of technology is considered as a positive occurence, recent factual and empirical evidence reveals that technology addiction augments and supplements a user's intrinsic and extrinsic gain perceptions about a system, leading to system overuse at a detrimental upraised level (Turel et al., 2011). Internet Addiction symptoms prevalent among technology addicts can cause negative personal, societal and workplace related consequences (Charlton \& Danforth, 2007). Excessive and uncontrolled Internet use is seen as a form of technological addiction which hits upon behavioral responses. Recent study has proved IA as a relatively recent and fast growing clinical phenomenon (Saville et al., 2010). This in turn has challenged the health parameters largely; China has reported that approximately 10 million citizens have scored high on Internet addiction tests (Block, 2008). Despite this growth, there are still many contradicting statements and beliefs in the field ranging from terminology and diagnosis to treatment.

There have been many negative social and psychological consequences, which has been the subject of scientific debate for many years, depending on the development of information technology and its availability. Addictive behavior is one of the forms of abnormal behavior, specifying a constant desire for a pleasant subjective emotional state, which is expressed in an active change in their mental state (Arakelyan, 2014; Arestova, Babanin, \& Voiskounsky, 2000; Bubnova \& Tereshchenko, 2016; Griffiths, 2001; Tang et al., 2014; Kardefelt-Winther, 2014). Some of the researchers also indicate maladaptive features which are impulsive and addictive in personality (Starcevic, 2013). Data also pathological internet use or problematic Internet use from $7.9 \%$ to $25.2 \%$ among adolescents (Ko et al., 2012), while the Middle-East and Africa had rates from 17.3\% to 23.6\%. Studies in parts of Asia have shown a higher variation in prevalence among young people and adolescents, ranging from $8.1 \%$ to $50.9 \%$ (Kim et al., 2015). In China, the rates ranged from 6\% to 10\% (Cao et al., 2011; Lai et al., 2013).

This was further proved by high-sensation seeking, loneliness and shyness, locus of control and online experience, attention-deficit/hyperactivity/ impulsivity symptoms (Fioravanti, Dèttore, \& Casale, 2012; Caplan, 2002; Caplan, 2003; Caplan, 2005; Kim et al., 2015). Also suicidal ideation an disordered psychological well-being (Kim et al., 2015), as well as the association with various personality traits (e.g.,Whang et al., 2003), interpersonal skills and intelligence. Purpose of this paper is to define terminology of Internet Addiction, present extent literature review on internet addiction, detailed available measurement and causes and consequences along with focusing on coping strategies from the pioneer researches to recent studies around the world.

\section{Defining Internet Addiction}

The first case study was given by Dr Kimberley Young, in the US, a serious research pioneer in this field who first published a case study regarding a 43-year old woman addicted to email (Young, 2000), further followed by the first 
seminal Internet addiction study (Young, 2000) which collected approximately 600 cases of people who suffered problems in their everyday life offline because they were unable to control Internet use. Silver and Aldrich in 1970 by highlighting that "changing technology has stimulated concerns that new electronic devices (e.g., radios) or the material transmitted via a new electronic device (e.g., dance music, jazz) cause addiction or disreputable behaviour". Europe also saw a similar initiative on internet addiction by Griffiths's paper on technological addictions, followed by a general paper on Internet addiction and specific aspects such as Internet gambling addiction. The term "addiction" has been criticized by many scientists also which has given emergence to less controversial terminologies describing the same phenomenon (Pezoa-Jares \& Espinoza-Luna, 2013). Currently, there is no consistent nomenclature with some of the different proposed terms given by many authors such as Internet dependency (teWildt, 2011), pathological Internet use (Morahan, 2000), problematic Internet use (Davis et al., 2002), compulsive computer use, etc. Many on the other hand contradicts with the internet as an additive medium, but use the Internet as a medium to fuel their specific addictions (Griffiths, 2001).

Therefore, there have been some different proposals about Internet Addiction classifications. Young (2000) view Internet Addiction as an umbrella term for a extensive variety of behaviors and impulse control problems that can be divided into five subtypes such as cyber sexual addiction, cyber-relationship addiction, net compulsions - obsessive online gambling, shopping or day-trading, compulsive web surfing, computer addiction - obsessive computer game playing. Alternatively, Davis (2001) proposes a theoretical cognitive and behavioral model of pathological Internet use (PIU) that differentiates between addictions to the Internet versus addictions on the Internet through two different characterizations one is Generalized Problematic Internet Use (GPIU) and the other is Specific Problematic Internet Use (SPIU).

Many study indicated that the term "Internet addiction" in general should be replaced by the respective addiction to a specific online activity when the behavioral addiction patterns for that activity are ascertained (Starcevic 2013). Cantelmi et al. (2000), keeping this in consideration proposed the term internet-related psychopathology (IRP) as the clinical conditions surrounding Internet Addiction are not an internet dependency, but a group of specific psychopathological situations in an online environment. Other suggested terms for the disorder are "internet-mediated psychopathology" (Tonioni 2013) and "internet spectrum dependency" (Karaiskos et al. 2010).

Ambiguity surrounding the acknowledged definition, term internet addiction was proposed by Dr. Ivan Goldberg for pathological compulsive internet use. Goldberg (1996) coined the term Internet Addiction Disorder (IAD) and gave clarification further to its definition as "the pathological, obsessive use of the Internet including tolerance and withdrawal as a standard for diagnosis". Young (2000) in his study defined pathological internet use (PIU) as "an impulse control disorder which does not involve an intoxicant or inebriant". Internet addiction is also defined as "a psychological dependence on the internet and is identified by a growing investment of resources on internet related activities, unpleasant and annoying feelings when offline, an increasing tolerance to the effects of being online, and denial of the controversial behaviors". Young (2000) defined pathological internet use (PIU) "as an intrinsic and deeprooted control disorder not including intoxicants". Stein (1997) described internet addiction as "uncontrollable and damaging use of the internet and is recognized as a compulsive impulsive internet usage disorder". Adding to it, studies considered internet addiction to be a "form of technological addiction and one of a subset of behavioral addictions such as compulsive gambling (Griffiths, 2001). Any behavior that shows the following six 'core components' of internet addiction such as salience, mood modification, tolerance, withdrawal symptoms, conflict, and relapse is operationally defined as functionally addictive by many researchers (Kim et al., 2015; Whang et al., 2003; Widyanto et al., 2011; Young, 2000).

A study in Korea by Kim et al. (2015) defined internet addiction as the use of the internet to such an extent as to cause emotional problems and dependent behavior indistinguishable to those of drug addiction. Shapira et al. (2000) defined controversial internet use by the following characteristics such as uncontrollable internet use, internet use which is markedly distressing, time consuming which results in social, occupational, or financial difficulties. Nalwa \& Anand (2003) in their study defined internet addiction as the psychological addiction to the internet and indicating as increasing investment to the activities on internet, unpleasant emotions when offline, increasing tolerance to the effect of become online and most importantly unacceptance of the problematic behavior showing themselves. A most recent definition by Kaplan and Sadock (2004) for addictive internet use (AIU) is described as dispensing with at least five of the six following benchmarks such as spending increasing amounts of time online, failure to cut back use with associated feelings of uneasiness and depression, staying online longer than requires or intended, running the risk of 
losing a relationship or other opportunity due to internet use, dishonesty in order to conceal or hide the extent of internet use, using the internet in order to move away from negative feelings.

Griffiths (2001) concludes referring to video games, computers, fruit machines-"There is little doubt that activities involving person-machine interactivity are here to stay and that such things as the interactive CDs and virtual reality consoles, the number of potential technological addictions (and addicts) will increase. To some people the notion of 'internet addiction' was first introduced by Dr. Kimberly Young in August 1996 at the Annual Meeting of The American Psychological Association (Young, 2000). The concept was highly debated by both clinicians and academicians especially on the use of the word 'Addiction'- as many believed that the term addiction should be used only to cases involving the ingestion of a drug. Young (1998) described the excessive and problematic use of the internet as an 'addictive disorder' and she is credited with coining the term 'Internet Addiction Disorder (IAD)', whereas other prefers Internet Addiction Disorder (Goldberg, 1996; Young, 2000), internet pathological use (Davis, 2001; Morahan-Martin and Schumacker, 2003), or Internet dependency (e.g., Scherer, 1997). Researchers have proposed the concept of internet addiction as an explanation for misuse or harmful use of internet accompanying the excessive or uncontrollable use of internet technology (Griffiths, 2001; Pratarelli et al. 1999; Kandell, 1998; Young, 2000).

\section{Measuring Internet Addiction}

There have been a myriad of instruments developed to assess Internet Addiction among a target population. Most of the instruments developed have not been verified and their psychometric properties have not been investigated within the context of different user groups or culture. As a matter of fact, in the most extensive and exhaustive literature review to date done by (Laconi et al., 2014) have identified almost 45 tools which measure and evaluate Internet addiction through either scales, interviews or diagnostic criteria. All the developed instruments given by Laconi are self-report questionnaires constructed using a theoretical basis and have no clinical validation. Only few instruments have reported criteria and/or cutoff points for Internet addiction although they are seldom based on empirical analyses. From all of these tools only 17 had their psychometric properties evaluated and assessed more than once, and only 10 had three or more evaluations. This largely implies that most of the reported scales require further scrutiny before they can be used by researchers and/or clinicians as measures for assessment of Internet Addiction.

The credibility of these scales is generally questionable mostly due to the lack of definition for Internet Addiction and its current grounding in multiple theoretical frameworks (Beard, 2005; Weinstein \& Lejoyeux, 2010; Lortie \& Guitton, 2013; Laconi et al., 2014). Other instruments for Internet Addiction evaluation The Internet Addiction Test (Young, 1998) is based on the Internet Addiction Diagnostic Questionnaire - IADQ (Young, 1998) and assesses Internet Addiction based on criteria for the diagnosis of pathological gambling. The measurement test broadly consists of 20 self-reported items scored on a 5-point Likert-type scale ranging from "rarely" to "always" permitting for a dimensional rather than categorical assessment. List of self-reported items include concepts such as loss of control, neglecting everyday life, behavioral and cognitive salience, negative consequences, mood modification, and deception. Based on these items users can be characterized as normal, having frequent problems or having significant problems. The modified Internet Addiction Test given by Young with a 6-point scale includes the "does not apply" option (Young, 2010) and differentiates between normal users and users with mild, moderate or severe Internet addiction. Other studies have shown an excellent test - retest reliability, $r>=0.75$, and excellent internal consistency, $a=0.92$ (Barke et al., 2012; Osada, 2013). Psychometric validations of the Internet Addiction Test have consistently resulted in differing dimensional structures, mostly dependent on culture, which strongly suggests that socio-cultural factors impact Internet addiction assessment (Widyanto et al., 2011).

Other measuring internet addiction tool, Compulsive Internet Use Scale - CIUS (Meerkerk et al., 2009) was developed primarily on criteria for pathological gambling, which included 14 items with a 5-point Likert-type scale, ranging from "never" to "very often". Furthermore, it has been validated in a 9-item form for adolescents (Cartierre et al. 2011) and it exists in a 17-item form (Guertler et al., 2014).

A tool developed to test among youth of Saudi Arabia, designed by Widyanto et al. (2011) has the constructs related to emotional/ psychological conflict, mood modification and time management, is considered and modifications are 
made to it. Internet Addiction Test is accepted by many researchers as a well-designed tool to measure the addiction level of individuals. There are various factors to validate the above, such as the first factor emotional/psychological conflict deals with the tendencies of the internet addicts like, low-self-esteem, social discomfort and others. The other factor is time management issues which brings out the factors that insist the person to spend time on the net related activities, which otherwise spent in productive manner. The last factor is mood modification which captures the psychological influences of the internet availability and deprivation like instable mental condition, depression and stress etcetera (Chen and Nath, 2016). Thus, multidimensional approach that is verified for its validity and reliability is the reason for the approval of this scale globally (Guofeng and Yuming, 2009; Khan and Ejike, 2017; Uwemi et al., 2016). Cultural factor, most significantly was added to the existing factors to know the impact of culture on the internet addiction tendencies along with the original three factors of Widyanto. Andreassen et al. (2012) recommended from their study about Bergen Work Addiction Scale substantiating every need to extend the research work towards exploring the impact of culture on internet addiction. A study done in China, Huang et al. (2007) mentioned that as per the social culture of Chinese population, internet chatting and internet communication is considered as the convenient and shielded way. Chen and Nath (2016) further added to it that the psychometric factors formulated by Widyanto change according to varied types of cultures. Thus, internet addiction and its relation with culture has a strong impact and influence on the mindset of the individuals and making them addicted (Eyadat, et al. 2012; Chang and Manlaw, 2008; Awan et al., 2016; Khan and Adediji, 2017).

Another measuring method was of Personality, MBTI (Mayers-Briggs Type Indicator), which has four directions of personality. The MBTI scale originated with Jung and it classified personality as introvert or extrovert and thinking or feeling (Briggs et al., 1999), in this study, classification of binary type questions each for introvert (vs. extrovert) and feeling-oriented (vs. thinking-oriented) tendency was formulated. The chosen number of introvert and feelingoriented examples were then added and converted to a six point personality scale, from 0 to 5 . Some other instruments are Short Internet Addiction Test (s-IAT)by (Pawlikowski et al., 2013; Griffiths, 2005). Brief Symptom Inventory subscale depression (Derogatis, 1983). Brief Symptom Inventory - subscale interpersonal sensitivity (Derogatis, 1983). Self-Esteem Scale (Collani and Herzberg, 2003) Self-Efficacy Scale (Schwarzer and Jerusalem, 1995). Trier Inventory for Chronic Stress (Schulz et al., 2004). Loneliness scale (Tilburg, 2006). Brief COPE (Carver, 1997). Internet Use Expectancies Scale (Velicer, 1976; Cohen, 1988).

\section{Causes of Internet Addiction}

Irresistible Internet use facilitates a psychological absconding mechanism to avoid the real problems. Considering that addictive personalities are more likely to suffer from negative thinking, which most likely in turn leads to low selfesteem and pessimistic attitudes (Hall \& Parsons, 2001). Also the anonymous interaction over the Internet upgrades these self-imposed deficiencies. This is dominantly evident in youth who 20 years ago used poetry poetry, music, and sports to express their needs and feelings, whereas now they turn to online activities to compensate their identity (Tao, 2005). This tendency to seek distraction leads a person to depart from an unpleasant and unwanted reality in order to create a virtual "ideal self" liberated from real-life stress and limitations ( $\mathrm{Li}$ et al. 2011). Hence, the factors leading to motivation for going online and spending unrestrained time have been explored and traversed in context of specific online activities. Studies shows that escapism is most clearly evident in online gaming platforms, where researchers have minutely investigated the relationship with internet addiction by exploring interaction effects between psychosocial problems and alleviating motivations for use.

Some studies focused the cause on the characteristic of anonymity in online environments people allow highly anxious individuals to compensate their loneliness by engaging in mutual gameplay. KardefeltWinther (2014) in the study brought out the relationship between stress and online gaming, which is mediated by the motivation for escapism. Other findings similar to this have been most importantly observed among players of massive multiplayer online roleplaying games (Zanetta-Dauriat et al. 2011, Lemenager et al. 2013). These findings further suggest it is necessary to further explore motivations for gaming and psychosocial well-being in conjunction.

Studies further proved from the social media perspective that expanding social networks to establish or enhance relationships is viewed as a tool to improving self-confidence, social abilities, and social support (Smahel et al., 2012). Individuals with high and uncontrolled level of social anxiety may perceive this form of communication a more 
acceptable form of interaction, due to the greater degree of control over one's image and also because of the lower risk of negative evaluation, which in turn may lead to Internet addiction (Lee \& Stapinski, 2012). The online environment urges individuals who struggle with identity crisis by giving them the opportunity to improve or change personas and frame their own identity in a favorable or sometimes unfavorable means. They use social networking to find psychological meaning to a profound and captivating requirement to feel emotionally close to others.

Among Other causes are Online Sexual Preoccupation, this can be caused by many of the Adult websites loaded with the sexual content for the purpose of arousing a sexual interest (Cooper, 1998).

Sexual addiction is also similar to gaming addiction which provides the Internet addicts with a room for imagination (Young, 2008). This usually refers to the activities of online viewing, downloading sexual materials, participating in the adult chat rooms, or uploading adult-content materials for the purposes of trading (Bianchi and Phillips, 2005; Brey,2006). Internet users who are addicted in this category, find this as an easy alternative to access sexually explicit materials while remain anonymous, thus are attracted into this form cyber addiction(Young,2004). Emailing is considered to be an effective communication tool in the emergence of mobile internet network (Joinson, 2003)

It is mostly considered in this generation that online environment gives the platform to express themselves and find acceptance missing in their lives. Another cause to the pathway of internet addiction may be body image problems and avoidance of real-life problems and real life interactions because of embarrassment relating to one's appearance (Rodgers et al., 2013).

Internet addiction can also result from consumption of online pornography due to its accessibility, affordability, and anonymity (Southern, 2008). Researchers view Internet addiction on a specter from a loosely controlled urge that causes anguish to a serious psychopathology (Young, 2009).

\section{Consequences of internet addiction}

The consequences of internet addiction have both positive and negative consequences. Positive perception stands that the individual feels better because of getting social stimulation and negative because he or she cannot make friends offline, proving to depend solely on the internet for social stimulation. This is internet addiction through pathological or clinical perspective. It is an understandable and practical way to acquire social stimulation when there is a lack of it (e.g., Chappell, Eatough, Davies, \& Griffiths, 2006; Leung, 2007; griffths, 2000), sometimes leading to negative consequences and addiction-like symptoms due to the amount of repayment required to alleviate negative feelings.

A study by Kardefelt-Winther (2014) proved psychosocial well-being was controlled for as the associations between psychosocial well-being or motivations for play and internet addiction may be false or fictitious. The ambiguity of its impact as well as its consequences both positive and negative in the social arena provokes researchers, from different areas of expertise, interest and attention to the problem (Arshinova \& Bartsalkina, 2010; Gubanov \& Chkhartishvili, 2009; Khil'ko, 2015; Khutornoi, 2013). Among the most discussed negative social and psychological consequences, the impact of the internet has had is the so called internet addiction phenomenon itself. Addictive behavior is one of the forms of abnormal behavior, distinguised by a constant desire for a pleasant subjective emotional state (Arakelyan, 2014; Arestova, Babanin, \& Voiskounsky, 2000; Bubnova \& Tereshchenko, 2016). Goldberg, a scientist from the US in the year 1996 first identified a new species in the classification of mental disorders - "cyber disorders" (Goldberg, 1996). It is the consequence of excessive stress, anxiety and fatigue as the results of long stay in the virtual world.

Psychological consequences is revealed in the works with specific flow experiences experienced by hackers known as psychological consequences of informatisation (Babaeva, Voiskounsky, and Smyslova, 2002; Kosenko, 2008; Asmolov, Tsvetkov, and Tsvetkova, 2004). Other consequences citing here are unhappy circumstances at home and at school may serve as both predictors and consequences of internet addiction A negative relationship with a teacher might cause extra stress, and adolescents might potentially use the Internet as a method to cope. A stranded relationship between parents and child is also streamed as one of the strongest consequences of internet addiction, further leading to risky behavior of the child (Mishna, Khoury-Kassabri, Gadalla, \& Daciuk, 2012; Yen et al., 2007). 
People who are addicted to internet can face the consequences that are similar to individuals who are addicted to alcohol (Lim et al., 2008), drugs (Romero et al., 2010; Bell et al., 2011), gambling (Walker, 1989), shopping (Pratarelli et al., 1999), sex (Carnes, 1999) or other irresistible behaviors (Greenfield, 1999). Many researchers also argued that a person's overuse or abuse of the internet is a behavioral demonstration of other things that may be problematic in their lives (Hayley, 2016). Egger (1996) in his study found a major difference between addicts and non-addicts, that addicts were significantly more likely to report negative consequences as a result of their Internet use; urges to use the Internet when off-line; feelings of guilt about the time spent on the internet (Scherer, 1997); telling giving false detail to their friends on the time they spent online (Kandell, 1998; Hinić, 2011) and complaints by colleagues about their excessive internet usage. Brenner's study (1997) indicated five internet overuse-related problems such as failure to manage time, lack of sleep, escaping meals, etc. Young's study (1998) found that excessive use of the Internet resulted in personal, family, and occupational problems similar to those experienced in other addictions i.e. missing sleep and meals. Healthy and unhealthy internet use stated that the internet use interfered with the academic work, professional performance, or social lives of 13.0 percent of the respondents was reported (Scherer, 1997). In many other studies, internet addiction is typically characterized by psychomotor agitation (Ahmet, 2012), anxiety (Shepherd and Edelmann, 2005) craving (Ferraro et al., 2007), depression (Young and Robert, 2009), hostility (Chiu and Huang, 2004), substance experience (Ko et al., 2014), preoccupation (LaRose et al., 2003), loss of control (Weinstein, 2010), withdrawal \{Griffiths, 2000; Seeman et al., (cited in Mitchell, 2000) and Bai, et al., (2000)\}, impairment of function (Hirschman, 1992; Larose et al., 2001; Wang, 2001), reduced decision-making ability (Ko et al., 2005), eating disorder (Lacey, 1993) and constant online surfing despite negative effects on social and psychological welfare (Hansen, 2002; Shaw and Black, 2008; Tao et al., 2010). Ashworth (1998), Burstenand Dombeck (2004), and Breedon (2009), Kang (1999) all have studies discussing the negative effects of internet addiction on our social lives. Studies of Clark et al., 2004; Murphy, 1996; Canbaz et al., 2009; Wang and Wang, 2008also revealed that heavy internet use for leisure was highly connected with impaired academic performance, particularly with those using synchronous communication applications like chat rooms and Multiple Users Dungeons (MUDs). Other common problems created by excessive internet use include disrupted marriages (Zahra et al., 2015; Armstrong et al., 2000; Hops et al., 1990), cyber sex addiction (Petrie and Gunn, 1998; Leung, 2003; Boies et al., 2004; Carnes, 2001; Harrill 2017), financial problems (Brenner, 1997; Lavin et al., 2000; Pratarelli et al., 2002), relationship problems (Lin et al., 2016; Penn, 2015; Ko et al., 2005; Putnam, 2000; Schneider, 2003; Young et al., 2000; Caplan, 2002; Kraut et al., 1998) and cyber relationship addiction (Parker and Benson, 2004; Cooper and Sportolari, 1997; Kaltiala-Heino et al., 2000; Shapiraet al., 2000).

\section{Coping strategies}

While internet addiction is not an officially recognized disorder, its critical and adverse effects on health and functioning has initiated an area of research focusing on treatment and prevention of internet addiction. All such addictions share specific characteristics and hence clinical interventions for internet addiction are based on therapeutic and pharmacological strategies that are most commonly used in conditions like OCD, impulse control disorders etc. the treatment itself consist of mainly psychotherapy, pharmacology or a combination of both, as these types of interventions were shown to be highly effective for decreasing the amount of time Internet addicts spent online, good at targeting symptoms of depression and anxiety (Winkler et al. 2013; King et al. 2011; Atmaca 2007; Przepiorka et al. 2014 ; Cash et al. 2012). Psychological treatment of internet addiction is focused around cognitive-behavioral therapy (CBT), considered as the most influential model (Young, 2013; Khazaal et al. 2012). In addition, CBT encourages the stimulation of activities which do not involve the use of the Internet in order to treat specific comorbidities (Cash et al. 2012; Young 2007). A study by 56 randomized Chinese adolescents divided into active treatment and waitlist control groups, only the active treatment groups demonstrated an improvement in time management skills and a decrease in emotional, cognitive, and behavioral symptoms post intervention (Du et al. 2010). Some study by researchers also describes combining psychological intervention on cognitive function and eventrelated potentials with electro-acupuncture as a treatment for patients with IA (Zhu et al., 2012; Young 2011). On the other hand other psychological treatments that might be beneficial are self-imposed bans on Internet access (Shaw \& Black 2008), abstinence programs (Kalke \& Raschke 2004), counseling programs (Shek et al. 2009) or multifamily group therapy (Liu et al. 2015; Ajdukoviü 1997). 
The psychological coping approach to internet addiction used in most studies consists of removing psychosocial vulnerabilities and problematic outcomes of internet use. Secondly bringing out a solution for Internet users high on social anxiety, and loneliness are at risk of neglecting schoolwork and resolving conflicts with parents due to their engagement with the internet. This paper suggests an inclusion of two additional elements that have been mentioned in this paper: the online activity and its affordances and discouraging for going online.

Allowing researchers to understand what and why the user is using the internet for and interpret the problematic outcomes against the background of the motivations for going online and the real life context of the user, enabling researchers to say something about why a person spends so much time online without resorting to speculation. Deeply exploring motivations in conjunction with psychosocial well-being allows us to elaborate on why someone goes online by contextualizing the motivation for excessive use in the presence of psychosocial problems.

\section{Concluding Remarks}

Since the emergence of the quantitative tools to scrutinize and assess the ramifications of internet usage both positively as well as negatively, there has been ambiguity of the resultant factors. It has been widely argued and largely accepted by the literature that many factors related to social, demographic, lifestyle changes related constructs have a bearing on the phenomenon strongly. Any kind of addiction destroys human beings physically, mentally, emotionally and psychologically. Internet addiction is no exception. The paper has discussed the status and various factors relating to internet addiction. Streams of literature proved that one of the most important findings is that internet addiction is closely related to personal factors such as the sense of alienation, powerlessness and isolation. Alienated consumers are compelled to resolve their anxiety through distorted behaviors, such as internet addictive consumption.

Addressing this problem, it is necessary to develop some remedies to lessen the addiction phenomenon. We need to develop effective consumer education programs to induce teenagers to use the internet effectively, offering them at school and home through online and offline mode.

Inducing in our education system in the course, by giving some alternative ways to teach students proper internet use.

Various family programs could be initiated which could be of great importance as the major internet usage place for addicts is basically the home. Various studies indicates that sound internet use within the family would surely improve the communication and interaction between family members. On the educational front it is highly necessary to lessen the burden on high-school students by reducing their stress and tensions level and reducing their sense of alienation and teaching them that internet use can actually increase these feelings. However, the role of education is very much established in both descriptive analysis and binary charts. As it is found that internet addiction is more prevailing among the people having higher and qualitative education than their counter parts, measures to safeguard the group against the unpropititios effect of internet addiction at local and global levels are the need of the hour. In addition to this, the factors like gender, age, income and education levels are able to explain the variation in the internet usage phenomenon. So, the results of the analysis lead to many theoretical and managerial implications. Also in-depth study and qualitative study with the addicts to know what is there in their mind is absolutely must be encouraged.

\section{References}

Arakelyan, L. K. (2014). The problem of the addiction in young people to social networks. Bulletin of the Council of young scientists and specialists, 1, 5-6.

Arestova, O. N., Babanin, L. N., \& Voiskounsky, A. E. (2000). The motivation of Internet users. Moscow: MozhaiskTerra.

Arshinova, V. V., \& Bartsalkina, V. V. (2010). Prevention of gambling on the internet, addiction in the educational environment. Moscow: MGPPU. 
Asmolov, A. G., Tsvetkov, N. A., \& Tsvetkova, A. V. (2004). Psychological model of Internet addiction personality. The World of Psychology, 1, 179-192.

Awan, M. A., Khan H.U., and Zhang, W. (2012). A comparative study on Online Service Quality Perception of two Major Regional Economies. International Journal of e-Education, eBusiness, e-Management and eLearning(IJEEEE), 2(6), 529-551.

Awan, M. A., Khan, H. U., \& Ho, H. C. (2016). Online Banking: A Comparative Study Of Chinese and Saudi Customers Perceptions Of Service Quality. Journal of Internet Banking and Commerce, 21(S5), 1-31.

Awan, M.A., and Khan, H.U. (2016). Status of Internet Addiction among College Students: A Case of South Korea. First American Academic Research Conference on Global Business, Economics, Finance and Social Sciences (AAR16 New York Conference), New York, USA, May 25- May 28, 2016. (Conference Proceeding).

Babaeva, J. D., Voiskounsky, A. E., \& Smyslova, O. V. (2002). The Internet: the impact on individuals. Moscow: Mozhaisk-Terra. 68-69

Barke, A., Nyenhuis, N., \& Kroner-Herwig, B. (2012). The German version of the internet addiction test: a validation study. Cyberpsychol Behav Soc Netw, 15, 34-542.

Beard, K.W., \& Wolf, E.M. (2001). Modification in the proposed diagnostic criteria for Internet addiction. Cyberpsychology Behav, 4, 377-383.

Beard, K.W. (2005). Internet addiction: a review of current assessment techniques and potential assessment questions. CyberPsychology Behav, 8, 7-14.

Billieux, J., Van Der Linden, M., Achab, S., Khazaal, Y., Paraskevopoulos, L., Zullino, D., \& Thorens, G. (2013). Why do you play World of Warcraft? An in-depth exploration of selfreported motivations to play online and ingame behaviours in the virtual world of Azeroth. Comput Human Behav, 29, 103-109.

Block, J.J. (2008). Issues for DSM V: Internet Addiction. Am J Psychiatry, 165, 306-307.

Bubnova, I. S., \& Tereshchenko, A. G. (2016). Prevention of addictive behavior among College students. Bulletin of Omsk University. Series: Psychology, 2, 4-11.

Burova, V. A. (2000). Internet addiction pathology of the XXI century? Scientific-practical journal. Issues of Mental Medicine and Ecology, 1(6), 11-13.

Cantelmi, T., Del Miglio, C., Talli, M., \& D'Andrea, A. (2000). Internetrelated psychopathology: first experimental data, clinical aspects and critical notes. Ital J Psychopathol, 6, 40-51.

Cao, H., Sun, Y., \& Wan, Y. (2011). Problematic Internet use in Chinese adolescents and its relation to psychosomatic symptoms and life satisfaction. BMC Public Health 11, 802. https://doi.org/10.1186/1471-2458-11-802

Chappell, D., Eatough, V., Davies, M.N.O., \& Griffiths, M. (2006). Ever Quest - It's Just a Computer Game Right? An Interpretative Phenomenological Analysis of Online Gaming Addiction. Int J Ment Health Addict, 4, 205-216.

Charlton, J.P., \& Danforth, I.D.W. (2007). Distinguishing addiction and high engagement in the context of online game playing. Comput Human Behav, 23, 1531-1548.

Derogatis, LR. (1983). SCL-90-R: Administration, scoring, and procedures manual II for the revised version. Towson, MD: Clinical Psychometric Research; 595-605.

Fioravanti, G., Primi, C., \& Casale, S. (2013). Psychometric evaluation of the Generalized Problematic Internet Use Scale 2 in an Italian sample. Cyberpsychol Behav Soc Netw, 16, 761-766.

Goldberg, I. (1996). Internet addictive disorder (IAD) diagnostic criteria. Retrieved from http://web.urz.uniheidelberg.de/Netzdienste/anleitung/wwwtips/8/addict.html

Greenfield, P. M. (2009). Technology and informal education: what is taught, what is learned. Science, 323(5910), 69- 71. https://doi.org/10.1126/science. 1167190

Griffiths, M. D. (2001). Sex on the Internet: Observations and implications for sex addiction. Journal of Sex Research, 38(4), 333-342. https://doi.org/10.1080/00224490109552104

Guan, S.S.A., \& Subrahmanyam, K. (2009). Youth Internet use: risks and opportunities. Curr Opin Psychiatry, 22, $351-356$.

Gubanov, D. A., \& Chkhartishvili, A. G. (2009). A model for information warfare in the social network. Management System and Information Technology, 3, 13-16.

Hinić, D. (2011). Problems with Internet addiction diagnosis and classification. Psychiatria, 2(23), 145-151.

Hall, A., \& Parsons, J. (2001). Internet addiction: College student case study using best practices in cognitive behavior therapy. J Ment Heal Couns, 23, 312-327.

Internet World Stats. (2012). Miniwatts. http://www.internetworldstats.com/stats.htm. Accessed 25 Aug 2015.

Kandell, J. J. (1998). Internet addiction on campus: The vulnerability of college students. CyberPsychology and Behavior, 1(1), 11-17. https://doi.org/10.1089/cpb.1998.1.11

Kardefelt-winther, D. (2014). Computers in Human Behavior A conceptual and methodological critique of internet addiction research: Towards a model of compensatory internet use. Comput Human Behav, 31, 351-354. 
Khil'ko, O. V. (2015). A practical approach to the prevention of computer addiction in adolescents. Scientificmethodical electronic journal "Concept", 13, 56-60. Retrieved from http://e-koncept.ru/2015/85012.htm

Khutornoi, S. N. (2013). Network virtual communication and its impact on interpersonal relationships. Modern Research of Social Problems, 6, 9-14.

Kim, H., Kim, Y.K., Gwak, A.R., Limm J.A., Lee, J.Y., Jung, H.Y., Sohn, B.K., Choi, S.W., Kim, D.J., Choi, J.S. (2015). Resting-state regional homogeneity as a biological marker for patients with Internet gaming disorder: A comparison with patients with alcohol use disorder and healthy controls. Prog Neuropsychopharmacol Biol Psychiatry, 60, 104-111.

Ko, C.H., Yen, J.Y., Chen, S.H., Wang, P.W., Chen, C.S., \& Yen, C.F. (2014). Evaluation of the diagnostic criteria of Internet gaming disorder in the DSM-5 among young adults in Taiwan. J Psychiatr Res.,53, 103-110.

Kosenko, I. I. (2008). The study of the Internet as a means of social communication. Informatics and Education, 9, 90 $-91$.

Lai, C.M., Mak, K.K., Watanabe, H., Ang, R.P., Pang, J.S., Ho. R.C. (2013). Psychometric properties of the IA test in Chinese adolescents. Journal of Pediatric Psychology, 38(7), 794-807.

Lee, B.W., \& Stapinski, L.A. (2012). Journal of Anxiety Disorders Seeking safety on the internet: Relationship between social anxiety and problematic internet use. J Anxiety Disord 2012, 26,197-205.

Lemenager, T., Gwodz, A., Richterm A., \& Reinhard, I. (2013). Selfconcept deficits in massively multiplayer online roleplaying games addiction. Eur Addict 2013.

Li, D., Liau, A., \& Khoo, A. (2011). Examining the influence of actual-ideal self-discrepancies, depression, and escapism, on pathological gaming among massively multiplayer online adolescent gamers. Cyberpsychol Behav Soc Netw, 14, 535-539.

Pezoa-Jares, R., \& Espinoza-Luna, I. (2013). Internet addiction: A review. J Addict, 12, 1-13.

Pontes, H., Szabo, A., \& Griffiths, M. (2015). The impact of Internetbased specific activities on the perceptions of Internet addiction, quality of life, and excessive usage: A crosssectional study. Addict Behav Reports 2015.

Pratarelli, M.E,, Brown,e B.L., \& Johnsonm K. (1999). The bits and bytes of computer/Internet addiction: a factor analytic approach. Behav Res Methods Instrum Comput. 31, 305-314.

Rodgers, R.F., Melioli, T., Laconim, S., Buim, E., \& Chabrol, H. (2013). Internet addiction symptoms, disordered eating, and body image avoidance. Cyberpsychol Behav Soc Netw, 16, 56-60.

Saville, B.K., Gisbert, A., Kopp, J., \& Telesco, C. (2010). Internet addiction and delay discounting in college students. Psychol Rec,60, 273-286.

Smahel, D., Brown, B.B., \& Blinka, L. (2012). Associations between online friendship and Internet addiction among adolescents and emerging adults. Dev Psychol, 48, 381-388.

Starcevic, V. (2013). Is internet addiction a useful concept? Spec Issue Aust New Zeal J Psychiatry,47, 16-19.

Tang, J., Yu, Y., Du, Y., Ma, Y., Zhang, D., and Wang, J. (2015). Prevalence of internet addiction and its association with stressful life events and psychological symptoms among adolescent internet users. Addictive Behaviors, 39 (3), 744-747.

Tao, H.K. (2005). Teenagers' Internet addiction and the qualityoriented education. J High Corresp Educ (Philosophy Soc Sci, 3, 70-73.

Turel, O., \& Serenko, A. (2010). Is Mobile Email Addiction Overlooked? Commun ACM, 53, 41-43. 123.

Weinstein, A., \& Lejoyeux, M. (2010). Internet addiction or excessive internet use. Am J Drug Alcohol Abuse, 36, $277-283$.

Whang, L.S., Lee, S., Chang, G. (2003). Internet over-users' psychological profiles: a behavior sampling analysis on internet addiction. Cyberpsychol Behav, 6, 143-150.

Widyanto, L., Griffiths, M.D., \& Brunsden, V. (2011). A psychometric comparison of the Internet Addiction Test, the Internet-Related Problem Scale, and self-diagnosis. Cyberpsychol Behav Soc Netw, 14, 141-149.

Young, K. (2000). Diagnosis - Internet-addiction. World Online, 2, 24-29.

Zanetta Dauriat, F., Zermatten, A., Billieux, J., Thorens, G., Bondolfi, G., Zullino, D., \& Khazaal, Y. (2011). Motivations to play specifically predict excessive involvement in massively multiplayer online role-playing games: Evidence from an online survey. Eur Addict Res, 17, 185-189. 